\title{
Force Feedback Enhancement for Soft Tissue Interaction Tasks in Cooperative Robotic Surgery
}

\author{
E. Beretta, F. Nessi, G. Ferrigno and E. De Momi
}

\begin{abstract}
Surgeons can benefit from the cooperation with a robotic assistant during the repetitive execution of precise targeting tasks on soft tissues, such as during brain cortex stimulation procedures in open-skull neurosurgery. Position-based force-to-motion control schemes may not be suitable solution to provide the manipulator with the high compliance desirable during guidance along wide trajectories. A new torque controller with non-linear force feedback (FFE) is presented to provide augmented haptic perception to the operator, during the instrument's placement on the tissue. The FFE controller was experimentally validated with a pool of non-expert users using brain-mimicking gelatin phantoms (8\% $-16 \%$ concentration). Besides providing hand tremor rejection for a stable holding of the tool, the FFE controller was proved to allow for a safer tissue contact with respect to both robotic assistance without force feedback and freehand executions $(50 \%$ and $\mathbf{7 5 \%}$ reduction of the indentation depth, respectively). Future work will address the evaluation of the safety features of the FFE controller with expert surgeons on a realistic brain phantom, also accounting for unpredictable tissue motions as during seizures due to cortex stimulation.
\end{abstract}

\section{INTRODUCTION}

Robotic technology can be used to supplement, augment, and improve human performance during the execution of manipulation tasks [1]. In particular, surgeons may benefit from robotic cooperation during the repetitive execution of precise targeting tasks on soft tissues, such as during brain cortex stimulation procedures in open-skull neurosurgery [2]. These tasks include the smooth motion of a tool (in the working area) and its accurate placement in a stable pose on the target tissues, thus requiring both an intuitive guidance during approaching and a safe and effective contact with tissue. Transparency, which quantifies the ability of a robot to cooperatively follow human movements without requiring any human-perceptible resistive force drive [3], is required in the target approaching phase, to reduce the fatigue of the user and the trajectory execution time. In the tool placement phase, the contact between the surgical instrument and the

E. Beretta is with Kuka Roboter GmbH, Zugspitzstraße 140, 86165 Augsburg, Germany (phone: +49 821797-5471; e-mail: elisa.beretta@kuka.com) and with the Electronics, Information and Bioengineering Department, Politecnico di Milano, p.zza Leonard da Vinci 32, 2013, Milano, Italy.

F. Nessi, G. Ferrigno and E. De Momi are with the Electronics, Information and Bioengineering Department, Politecnico di Milano, p.zza Leonard da Vinci 32, 20133, Milano, Italy (e-mail: federico.nessi@polimi.it, elena.demomi@polimi.it; giancarlo.ferrigno@polimi.it). underneath tissue must be guaranteed stable, filtering hand tremors, and safe, preventing any damage to the tissue due to indentation. Also, if predictable and/or unpredictable motions of the soft organ of interest occurs, e.g. respectively due to breathing and pulsatility [4] and/or to epileptic seizures during brain cortex stimulation in neurosurgery [5], the robotic assistant should be able to compensate them and react safely.

Touch interactions and physical contacts are critical factors during the manipulation of tissue/objects. Haptic feedback in tele-operation and cooperative manipulation systems has been an active area of research for several decades, although its benefits in robotic surgery have not yet been assessed in terms of clinical outcome [6] and major issues have prevented its use in a commercially available system [7][8], e.g. the intrinsic trade-off between stability and transparency and the challenge of sensing forces under cost, biocompatibility and sterilizability constraints. However, force feedback was proved to allow for tissue characterization [7][9], lower applied forces on tissues [10] and reduced unintentional injuries [11]. In particular, position-based impedance controlled (admittance) assistants, guided by the instant feedback of forces measured at the surgical instrument, prevent force-induced damage to soft tissues and reduce the surgeon fatigue both for tele-operated devices, e.g. in heart surgery [12], and for cooperative manipulation systems, e.g. in vitro-retinal surgery [13] and in assisted needle insertion in keyhole neurosurgery [8]. However, this approach is not advisable for surgical targeting tasks, which require the repetitive execution of wide maneuvers from/to a home robotic configuration, e.g. during brain mapping procedures.

In this context, a highly compliant cooperative manipulator is desirable to promptly react to the applied forces without resisting to the surgeon's guidance, thus a torque-based impedance controller is more suited [14]. Impedance-controlled cooperative robots, e.g. the Acrobot system with back-drivable joints for robotic assisted orthopaedic surgery [15], allow the natural transmission to the user of the interaction forces with the environment, both for human guidance and/or for tissue contact, without requiring direct force sensing [16]. Nevertheless, an enhancement of the tool-tissue kinaesthetic information is needed to provide haptic sensation to the user, due to the small ranges of forces involved in the interaction with soft tissues.

In this work, we propose to cooperatively assist surgical targeting task on soft tissues with a non-linear force feedback torque control, in order to augment the surgeon's skills during the placement of the surgical instrument with respect to pure visual feedback. The performance of the robotic assistance controller was assessed on brain-mimicking gelatin phantoms, which were mechanically characterized to 
quantitatively evaluate the tissue damage due to the contact with the tool, thus defining a safety criterion on the maximum force and penetration allowed during the indentation. The performances of the robotic assistance with and without force feedback augmentation were comparatively evaluated with respect to freehand task executions, both in terms of effectiveness in preventing the insertion of the surgical tool during the target approaching and of position/force tremor rejection during the holding phase.

The paper is organized as follows: related work is summarized in Section II; the proposed enhanced force feedback loop is presented in Section III, together with the experimental evaluation on brain-mimicking phantoms; results are presented in Section IV and discussed in Section V.

\section{RELATED WORK}

Cooperative manipulation systems based on admittance control proved to be very effective in highly demanding surgical procedures requiring high tool positional accuracy, e.g. vitro-retinal surgery [13][17] and keyhole neurosurgery [8], thus where a "very stiff" dynamic behavior of the robotic device is desirable. Scaled force reflection strategies [13][18] were developed for the John Hopkins "Steady-Hand Eye" robot in vitro-retinal surgery, where surgeons are required to perform micro scale maneuvers while safely applying forces that are below sensory perception. In particular, a micro-force guided cooperative control, enforcing a global limit on the forces applied at the robot tool tip and actively guiding the operator towards the direction of lower resistance, proved to be effective in enforcing force limits during peeling tasks [13]. Also, sclera contact location and force measurements were recently used to adapt the remote center-of-motion (RCM) constrain in a variable admittance controller in order to minimize the eye motion while enabling tool manipulation inside the eye, as well as provide useful sclera force feedback to assist to reposition the eye [17]. Position-based force-tomotion control schema with linear force feedback enhancement were implemented also for cooperative assisted needle insertion devices [8], thus providing the user with the haptic feeling required to detect changing tissue properties of subsurface structures at different depths. All the abovementioned force tracking control approaches exploit the manipulator with high stiffness dynamics and suffer from the inability to provide a "soft" compliant behavior [14].

Torque-based impedance control is better suited to provide the small stiffness and damping desirable in reducing contact forces [14]. Different strategies of variable impedance controller were proposed to adapt the dynamics of the manipulator during free-motion guidance for surgical [19], industrial [20] and assistive robotics [21] applications. Among the different strategies presented to modulate the impedance of the manipulator, we previously proposed and tested a variable damping controller based on the robotic endeffector position [22], which was proved to be appropriate for the aforementioned surgical scenario, due to the varying accuracy and safety requirements depending on the patient position in the operating theater. However, robotic mechanical impedance may mask any delicate force arising from the interaction with soft tissues [23], reducing the user perceivable level [24]. In particular, interaction forces during indentation on swine brain tissue were estimated below $0.2 \mathrm{~N}$ with a $10 \mathrm{~mm}$ diameter indentor pushed at a depth of $10 \mathrm{~mm}$ [25]. The maximum peak forces during manual and robotic direct assess needle insertion respectively into in-vivo bovine liver and kidney were reported in the range 0.7-0.8N [26].

\section{MATERIALS AND METHODS}

\section{A. Torque control with force feedback enhancement}

In order to provide a "soft" dynamic behavior during the cooperative guidance [14], the torque control for flexible joint manipulators reported in [16] is used. As shown in Fig. 1 , the torque input of the joint state feedback controller $(\boldsymbol{\tau})$ is computed as:

$$
\tau=\tau_{d}-\widehat{\tau}_{n}+\tau_{e}
$$

where $\boldsymbol{\tau}_{d}$ is the desired torque vector, $\hat{\boldsymbol{\tau}}_{n}$ is the feed forward model-based torque vector for the compensation of the robot's dynamic, i.e. gravity and Coriolis-centrifugal terms, and $\tau_{e}$ is the vector of the external torques applied to the manipulator by the environment. For our study, we assume two different types of environmental interaction $\left(\mathbf{f}_{e}\right)$ :

- Human interaction forces $\left(\mathbf{f}_{h}\right)$ : the operator guides

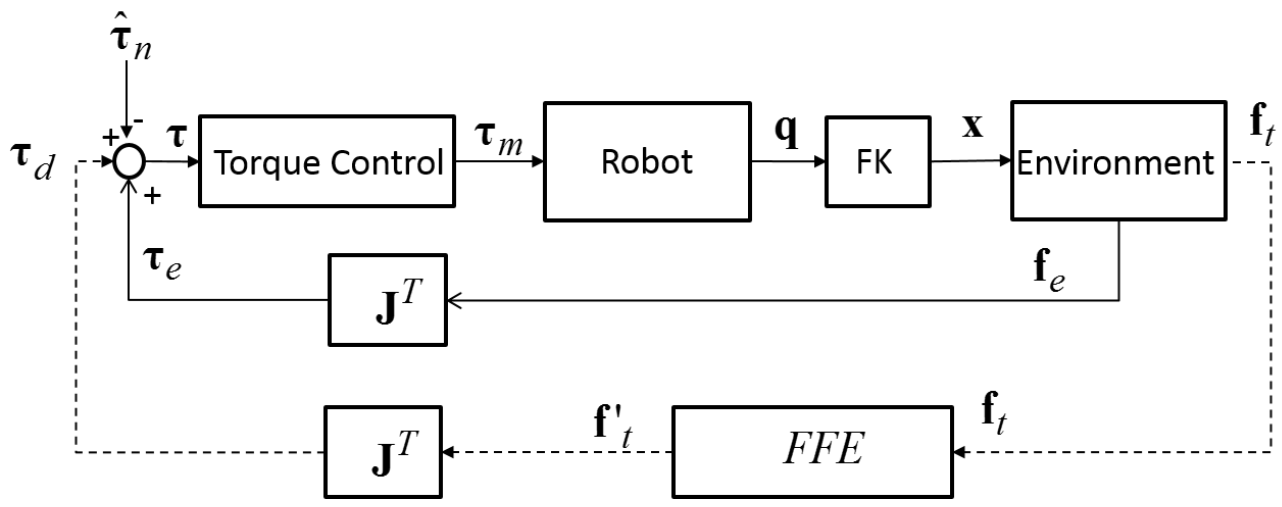

Figure 1. Torque control with force feedback enhancement (FFE). $\boldsymbol{\tau}_{m}$ is the motor torque vector, $\boldsymbol{\tau}$ is the joint torque vector, $\mathbf{q}$ is the joint configuration vector and $\mathbf{x}$ is the Cartesian end-effector position computed with the Forward Kinematic (FK) block. The environment forces ( $\mathbf{f}_{\boldsymbol{e}}$ ) represents both the operator's hand and the tissue during the cooperative guidance. The tissue contact forces $\left(\mathbf{f}_{t}\right)$ are directly measured with a force sensor on the instrument and the desired torque vector $\left(\boldsymbol{\tau}_{d}\right)$ is computed according to the enhancement strategy (dot line). 


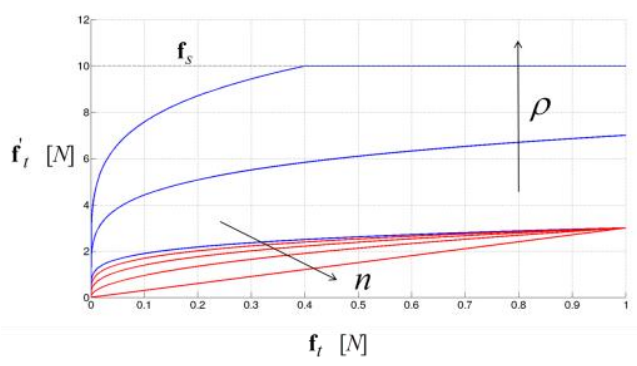

Figure 2. Non-linear force feedback enhancement parameters.

the manipulator applying external forces with a single-point contact during the free-motion guidance phase and the instrument's placement phase, depending on his/her unknown intention of motion [27];

- Soft tissue interaction forces $\left(\mathbf{f}_{t}\right)$ : the compression /indentation reaction of the soft tissue during the instrument's placement phase depends on its viscoelastic mechanical properties [28].

Thus, the external torque vector can be written as:

$$
\boldsymbol{\tau}_{e}=J^{T}\left(\mathbf{f}_{h}+\mathbf{f}_{t}\right)+\boldsymbol{\tau}_{n}
$$

where $J$ is the Jacobian matrix of the manipulator and $\boldsymbol{\tau}_{n}$ represents the effects of the robot dynamics. Non-idealities of the robot dynamic models $\left(\widehat{\tau}_{n} \neq-\tau_{n}\right)$ limit the maximum transparency of the system [27]. In fact, during the target placement phase, which can be assumed as a quasi-static scenario, the residual friction of the manipulator may mask the delicate interaction forces generated by the contact with the soft tissue. Thus, a force feedback enhancement loop is required to provide haptic perception to the user during tissue indentation. The robotic system is provided with a force sensor placed on the instrument to allow the direct measurements of the soft tissue interaction forces $\left(\mathbf{f}_{t}\right)$ and the non-linear force feedback enhancement (FFE) is computed as:

$$
\mathbf{f}^{\prime} t_{t}=\left\{\begin{array}{cc}
\rho\left(\mathbf{f}_{t}\right)^{n} & \left\|\mathbf{f}^{\prime} t\right\|<\mathbf{f}_{s} \\
\mathbf{f}_{s} & \left\|\mathbf{f}^{\prime} t^{\prime}\right\| \geq \mathbf{f}_{s}
\end{array}\right.
$$

where $\rho$ is a positive scalar $(\rho>1)$ and $n$ is the positive exponent of the polynomial function in the range $0-1$, thus enhancing the contrast for small forces (Fig. 2). A saturation level $\mathbf{f}_{s}$ defines the maximum force feedback perceivable from the tissue, thus representing the maximum force the user has to overcome to intentionally penetrate the tissue. The commanded torque vector is then computed as:

$$
\boldsymbol{\tau}_{d}=J^{T}\left(\mathbf{f}_{t}^{\prime}\right)
$$

Thus, the balance of forces at the contact point, assuming $\widehat{\tau}_{n}=-\tau_{n}$, results when:

$$
\begin{aligned}
& J^{T}\left(\mathbf{f}_{t}^{\prime}+\mathbf{f}_{t}+\mathbf{f}_{h}\right)=0 \\
& \mathbf{f}_{h}=-\left(\mathbf{f}^{\prime}{ }_{t}+\mathbf{f}_{t}\right) \approx-\rho\left(\mathbf{f}_{t}\right)^{n}
\end{aligned}
$$

considering the tissue interaction forces negligible with respect to the augmented forces.

The stability of the proposed feedback controller was evaluated through a passivity analysis to verify if the energy flow in a system is dissipative. Assuming that the guidance and tissue interaction forces are applied on a single control point ( $\mathbf{x})$, the passivity is guaranteed imposing the following condition:

$$
\int_{0}^{t} \dot{\mathbf{x}}\left(\mathbf{f}_{h}-\rho\left(\mathbf{f}_{t}\right)^{n}\right) d t>-E(0)
$$

where $E(0)$ is the energy of the system stored in time $\mathrm{t}=0$. The position of the manipulator is modeled according to distance from the surface of the soft tissue $(x)$, where positive values indicate tissue indentation. Negative values (motion in free space) would not be considered. The steady state initial condition is defined at null indentation level ( $\dot{x}(0)=x(0)=0, E(0)=0)$ with a step input signal of the human force $\left(f_{h}(0)=\bar{f}_{h}\right)$. Assuming an ideal elastic model of the tissue with constant stiffness $(K)$, at the equilibrium ( $\left.f_{h}=\rho\left(f_{t}\right)^{n}\right)$ the human force input can be expressed according to the soft tissue reaction as:

$$
\bar{f}_{h}=\rho(K \bar{x})^{n}
$$

where $\bar{x}$ indicates the indentation level at the equilibrium.

The passivity condition (7) resulted in:

$$
x<\sqrt[n]{(n+1)} \cdot \bar{x}
$$

and thus is always verified for positive values of $n$.

\section{B. Experimental Protocol}

The proposed control approach was experimentally evaluated using the LWR4+ (Kuka, Augsburg, Germany), a 7 degrees-of-freedom (DoFs) flexible joint manipulator, which features $0.05 \mathrm{~mm}$ repeatability (as per datasheet) and $\approx 1 \mathrm{~mm}$ accuracy [29]. Joint torque sensors allow the measurements of external torques along the manipulator's structure $\left(\tau_{e}\right)$. In order to distinguish between the interaction forces due to the human guidance and to the soft tissue indentation, a 6 DoFs Nano43 F/T sensor (ATI Industrial Automation, Apex, NC), which features $1 / 512 \mathrm{~N}-1 / 40 \mathrm{Nmm}$ resolution in a $9 \mathrm{~N}$ $125 \mathrm{Nmm}$ range, was mounted between the robot flange and a linear tool ( $3 \mathrm{~mm}$ diameter tip), mimicking the coaxial surgical tool for brain stimulation (Fig. 3). In order to compare between free-hand and robotic assisted task executions, the Gamma F/T sensor (ATI Industrial Automation, Apex, NC), which features $1 / 80 \mathrm{~N}-1 / 2 \mathrm{Nmm}$ resolution in a $32 \mathrm{~N}-2.5 \mathrm{Nm}$ range, was also used as a load cell to measure the interaction forces on the soft material's phantoms. A 3D-printed horizontal calibration plate with two orthogonal axis $\left(\mathbf{R F}_{\mathrm{P}}\right)$ was rigidly fixed to the load cell. The Certus optical localization system (Northern Digital Instruments, Ontario, Canada) with active markers $(0.1 \mathrm{~mm}$ accuracy) was used to track the instrument pose $\left(\mathbf{R F}_{\mathrm{EE}}\right)$ and the calibration plate $\left(\mathbf{R F}_{\mathrm{CP}}\right)$ in the camera frame $\left(\mathbf{R F}_{\mathrm{C}}\right)$. A reference frame with the origin coincident with the tip of the 


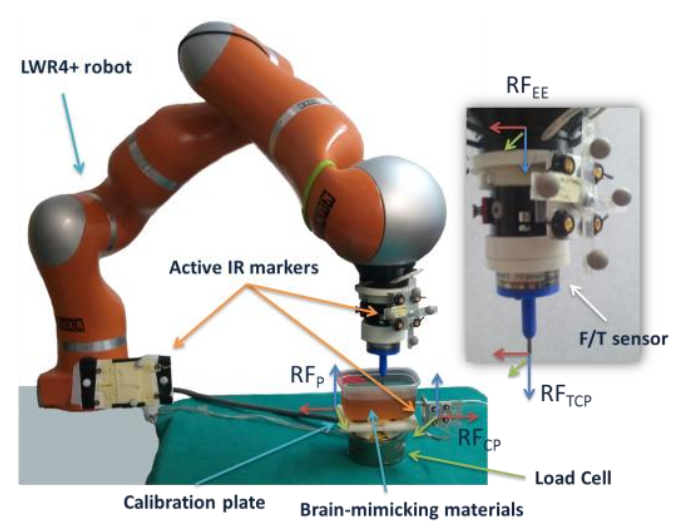

Figure 3. The experimental setup. The reference frames (RFs) of interest are also reported

linear tool and the z-axis coaxial with the tool's principal axes $\left(\mathbf{R F}_{\mathrm{TCP}}\right)$ was defined with respect to the end effector reference frame $\left(\mathbf{R} \mathbf{F}_{\mathrm{EE}}\right)$ using a pivoting procedure. Using a hand-eye calibration procedure, the tool tip position $\left(t_{\mathrm{P}}\right)$ and the force measurements of the end-effector sensor $\left(f_{\mathrm{P}}\right)$ and of the load cell $\left(l_{\mathrm{P}}\right)$ were computed in $\mathbf{R F}_{\mathrm{P}}$ (using the CAD models of the printed plate).

Tests were performed mimicking the target approaching gestures of an open-skull neurosurgical procedure using gelatin phantoms with three different concentrations $(8,12$, $16 \%$ ), that mimic the viscoelastic characteristics of the brain tissues [30]. The flat surface of each phantom was calibrated in the robot base reference frame with a least square regression process on 12 points acquired with an optical pointer (root mean square error $<0.5 \mathrm{~mm}$ ).

The robotic control system was implemented in the OROCOS and ROS frameworks (www.orocos.org, www.ros.org) and controlled in a real-time environment guaranteed by a Xenomai patched kernel (www.xenomai.org). Torque commands were computed at 1 $\mathrm{kHz}$ [16], while the F/T signals were acquired at $2 \mathrm{kHz}$. The end-effector $\mathrm{F} / \mathrm{T}$ measurements were processed with a notch filter (cut frequency $186.5 \mathrm{~Hz}$, minimum order) to cancel out the noise derived from the robot's fan vibrations. Also, the dynamic of the linear tool was online compensated [31], resulting in a $0.02 \mathrm{~N}$ accuracy of the force measurements at the end-effector.

The experimental protocol included three phases:

1) tissue characterization for contact damage quantification;

2) optimization of the FFE controller;

3) evaluation of the FFE controller performance.

\section{1) Tissue characterization for contact damage quantification}

During brain stimulation the surgeon places the stimulators in contact with the brain cortex. Large levels of indentation, up to possible penetration, may cause tissue damage. In order to quantitatively define in which range of forces penetration occurs, thus identifying a safety criterion for the gelatin indentation, the gelatin phantoms were characterized in terms of puncturing force $\left(\overline{\mathbf{f}}_{t}\right)$ and penetration level $\left(\bar{p}_{t}\right)$. The protocol included 8 trials of autonomous robotic tool insertions at three different velocities $(4-16 \mathrm{~mm} / \mathrm{s})$ performed for each gelatin at different concentrations $(8-16 \%)$. Each gelatin phantom was kept for at least 8 hours at a temperature of $5^{\circ} \mathrm{C}$ before use and changed at every experiment to avoid degradation of the material due to the environmental temperature $\left(25^{\circ} \mathrm{C}\right)$. The $\mathrm{F} / \mathrm{T}$ signals from the end-effector sensor $\left(f s_{\mathrm{P}}\right)$ were processed with a first order adaptive filter [32] to identify the peak related to the puncturing event. The median value and the first and third Inter Quartile Ranges (IQR 25\%; IQR 75\%) of the puncturing forces and the correspondent penetration level among trials are reported in Table I. A safety level for each different gelatin concentration is identified according to the minimum puncturing force $(0.4 \mathrm{~N}$ with $8 \%, 0.7 \mathrm{~N}$ with $12 \%$, $1 \mathrm{~N}$ with $16 \%$ ).

\section{2) Optimization of the FFE controller}

The force feedback enhancement parameters $(n, \rho)$ were optimized considering the critical tool insertion scenario $(8 \%$ gelatin at $4 \mathrm{~mm} / \mathrm{s})$, which reports the minimal puncturing force (mean value $0.41 \mathrm{~N}$ at $\approx 5.5 \mathrm{~mm}$ mean penetration) (Table I). Due to the residual static friction of the system $( \pm 1.5 \mathrm{Nm}$ internal thresholds on the robot joint torque sensors), the minimum user perceivable force $\left(\underline{\mathbf{f}}_{h}\right)$ during assisted targeting tasks is strictly related to the static friction of the system $(\approx 2 / 3 \mathrm{~N})$ and was considered equal to $4 \mathrm{~N}$. The optimal nonlinear characteristic $(n=0.8)$ was computed in order to limit the propagation of the measurement error of the force sensor $(0.02 \mathrm{~N})$ below $20 \%$ of $\underline{\mathbf{f}}_{h}$. Also, the amplitude parameter $(\rho)$ was tuned so that the user was able to perceive contact when the tissue reacts with a force equal to a percentage $(\lambda=50 \%)$ of the critical puncturing force as:

$$
\rho=\frac{\underline{\mathbf{f}}_{h}}{\left(\lambda \overline{\mathbf{f}}_{t}\right)^{n}} \approx 14.5
$$

The saturation force $\mathbf{f}_{s}$ was set equal to $10 \mathrm{~N}$.

\section{3) Evaluation of the FFE controller performance}

During targeting an arbitrary point of the gelatin surface was approached and the pose was held for $2 \mathrm{~s}$. The users were asked to keep contact with the tissue during the interaction, preventing unwanted gelatin damage. The effectiveness of the proposed FFE loop was comparatively assessed during repetitive targeting tasks on a pool of 6 non-expert users. Every user performed 8 targeting gestures in a randomized order on $8 \%, 12 \%$ and $16 \%$ gelatin samples in these

TABLE I. PUNCTURING FORCES $\left(\overline{\mathbf{f}}_{t}\right)$ AND DEPTHS $\left(\bar{p}_{t}\right)$ AMONG GELATIN CONCENTRATIONS AND TOOL INSERTION VELOCITIES

\begin{tabular}{|c|c|c|c|c|}
\hline \multirow{2}{*}{$\begin{array}{c}\overline{\mathbf{f}}_{t} / \bar{p}_{t} \\
{[\mathrm{~N}] /[\mathrm{mm}]}\end{array}$} & \multicolumn{4}{|c|}{ Indentation Velocities $[\mathrm{mm} / \mathrm{s}]$} \\
\hline & 4 & 8 & 12 & 16 \\
\hline $8 \%$ & $\begin{array}{c}0.41 \pm 0.02 * / \\
5.5 \pm 0.3\end{array}$ & $\begin{array}{c}0.50 \pm 0.02 / \\
6.3 \pm 0.4\end{array}$ & $\begin{array}{c}0.52 \pm 0.01 / \\
6.4 \pm 0.2\end{array}$ & $\begin{array}{c}0.57 \pm 0.03 / \\
6.3 \pm 0.4\end{array}$ \\
\hline $12 \%$ & $\begin{array}{c}0.78 \pm 0.02 / \\
5.3 \pm 0.1\end{array}$ & $\begin{array}{c}0.96 \pm 0.03 / \\
6.1 \pm 0.2\end{array}$ & $\begin{array}{c}1.08 \pm 0.01 / \\
6.4 \pm 0.1\end{array}$ & $\begin{array}{c}1.14 \pm 0.05 / \\
6.5 \pm 0.4\end{array}$ \\
\hline $16 \%$ & $\begin{array}{c}1.04 \pm 0.03 / \\
5.5 \pm 0.2\end{array}$ & $\begin{array}{c}1.27 \pm 0.05 / \\
6.4 \pm 0.3\end{array}$ & $\begin{array}{c}1.42 \pm 0.03 / \\
6.7 \pm 0.2\end{array}$ & $\begin{array}{c}1.44 \pm 0.04 / \\
6.7 \pm 0.1\end{array}$ \\
\hline
\end{tabular}


modalities:

- Freehand $(\mathrm{FH})$ : the targeting task was performed with an optical pointer (3mm diameter tip); the pencil-style hand grip is equal to the standard bipolar stimulator tools;

- Robotic assistance (RA) without force feedback;

- Robotic assistance with force feedback enhancement loop (FFE).

In both the robotic assistive cases (RA and FFE), the user performed the targeting tasks guiding the 3 translational DoFs of the manipulator, while the orientation of the tool was fixed to an initial configuration. During each trial, the force and TCP position signals were processed, in order to detect the beginning/end of the indentation phase and of the holding phase during the tool-tissue contact. The following indexes of performance were evaluated for each trial:

- Execution time (T): computed from the moment in which the TCP is close $5 \mathrm{~cm}$ to the surface to the identified initial time of the holding phase;

- Mean equilibrium force $\left(\mathbf{F}_{\mathrm{H}}\right)$ : computed as the mean norm force value from the load cell measurements between time samples;

- Mean penetration level $\left(\mathbf{P}_{\mathrm{H}}\right)$ : computed as the mean signed distance from the registered surface between time samples (positive values refers to position below the surface, thus indentation depths);

- Amplitude variability of the force/penetration $\left(\mathbf{V}_{\mathrm{F}}\right.$ $/ \mathbf{V}_{\mathrm{P}}$ ): computed as the root mean square (RMS) distance from the mean values $\left(\mathbf{F}_{\mathrm{H}}\right.$ and $\mathbf{P}_{\mathrm{H}}$ respectively) along time samples.

The median value and the first and third IQR were computed over different trials and different users for $\mathbf{T}, \mathbf{F}_{\mathrm{H}}$ and $\mathbf{P}_{\mathrm{H}}$ indexes. A comparative analysis of the different task execution modalities (FH, RA, FFE) and of the different tissue mechanical properties (8-16\% gelatin concentration) was carried out using the Kruskal-Wallis test with Bonferroni-Holms correction $(\mathrm{p}<0.05)$. The amplitude variability indexes $\left(\mathbf{V}_{\mathrm{F}}\right.$ and $\left.\mathbf{V}_{\mathrm{P}}\right)$ were computed over trials of each user in each execution modality (FH, RA, FFE) and for each gelatin concentration $(8 \%, 12 \%, 16 \%)$. For counting statistics, a sample distribution was measured under the hypothesis that the amplitude variability in freehand modality is greater than in the other assisted cases. The amplitude variability indexes among users were also computed.

\section{RESULTS}

The analysis performed on the execution time (T) index is reported in Table II. The execution time computed for the RA (mean time below 3s) and FFE (mean time below 2.5s) modalities resulted significantly greater than the one required to execute the task in FH mode (mean time below 1.3s) for each experimental conditions. No statistically significant difference was reported between the RA and FFE modes.

The results of the comparative analysis performed on the mean equilibrium force $\left(\mathbf{F}_{\mathrm{H}}\right)$ and penetration $\left(\mathbf{P}_{\mathrm{H}}\right)$ indexes during the holding phase are reported in Figure 5. The mean
TABLE II. EXECUTION Time (T) AMONG MODES AND GELATIN CONCENTRATIONS

\begin{tabular}{|c|c|c|c|}
\hline $\mathbf{T}[\mathbf{s}]$ & $\boldsymbol{F H}$ & $\boldsymbol{R A}$ & $\boldsymbol{F F E}$ \\
\hline $\mathbf{8 \%}$ & $1.3 \pm[0.6 ; 0.3]^{*}$ & $2.7 \pm[0.9 ; 0.8]$ & $2.5 \pm[0.8 ; 1.7]$ \\
\hline $\mathbf{1 2 \%}$ & $0.8 \pm[0.4 ; 0.5]^{*}$ & $2.9 \pm[1.2 ; 1.5]$ & $2.0 \pm[0.7 ; 1.1]$ \\
\hline $\mathbf{1 6 \%}$ & $0.7 \pm[0.4 ; 0.4]^{*}$ & $2.6 \pm[0.5 ; 0.7]$ & $2.4 \pm[0.6 ; 1.5]$ \\
\hline
\end{tabular}

(*) statistically significant difference among task execution modalities

TABLE III. AMPLITUDE VARIABILITY OF PENETRATION $\left(\mathbf{V}_{\mathbf{P}}\right)$ AND FORCE $\left(\mathbf{V}_{\mathbf{F}}\right)$ AMONG MODES AND GELATIN CONCENTRATIONS

\begin{tabular}{|c|c|c|c|}
\hline $\begin{array}{c}\mathbf{V}_{\mathbf{F}}[\mathrm{N}] / \\
\mathbf{V}_{\mathrm{P}}[\mathrm{mm}]\end{array}$ & $\boldsymbol{F H}$ & $\boldsymbol{R A}$ & $\boldsymbol{F F E}$ \\
\hline $\mathbf{8 \%}$ & $0.04 * / 0.8^{*}$ & $<0.02 /<0.1$ & $<0.02 /<0.1$ \\
\hline $\mathbf{1 2 \%}$ & $0.06^{*} / 0.6^{*}$ & $<0.02 /<0.1$ & $<0.02 /<0.1$ \\
\hline $\mathbf{1 6 \%}$ & $0.13^{*} / 0.6^{*}$ & $<0.02 /<0.1$ & $<0.02 /<0.1$ \\
\hline
\end{tabular}

$\left.{ }^{*}\right)$ the assumption $\left(\mathbf{V}^{\mathrm{FH}}>\mathbf{V}^{\mathrm{RA}}\right)$ and $\left(\mathbf{V}^{\mathrm{FH}}>\mathbf{V}^{\mathrm{FFE}}\right)$ is verified among trials and users

equilibrium forces $\mathbf{F}_{\mathrm{H}}$ was significantly smaller when the task is executed in FFE mode (75\% quartile value below the $0.2 \mathrm{~N}$ perception threshold) with respect to both FH and RA modes with all the gelatin phantoms. While the comparable equilibrium forces computed in FH and RA modes significantly increased according to higher gelatin concentrations, no statistically significant difference was computed between the $12 \%$ and $16 \%$ gelatin phantoms in FFE mode (mean value $<0.1 \mathrm{~N}$ ). The $\mathbf{F}_{\mathrm{H}}$ indexes resulted below the identified safety level for the different gelatin concentration $(0.4 \mathrm{~N}$ with $8 \%, 0.7 \mathrm{~N}$ with $12 \%, 1 \mathrm{~N}$ with $16 \%)$.

Accordingly, the penetration depth at the equilibrium point $\left(\mathbf{P}_{\mathrm{H}}\right)$ during $\mathrm{FH}$ task executions (mean value above $1.5 \mathrm{~mm})$ resulted significantly greater with respect to RA (mean depth in the range $0.5-1 \mathrm{~mm}$ ) and FFE (mean depth below $0.6 \mathrm{~mm}$ ) modes. No statistically significant differences are computed among the penetration depths on gelatin phantoms with different concentrations both for the $\mathrm{FH}$ (except for the comparison between $8 \%$ and $12 \%$ gelatins) and RA modes. Conversely, the penetration depths on $16 \%$ gelatin during FFE task executions resulted significantly smaller than the other cases. In this case, the depth values are below the $0.5 \mathrm{~mm}$ surface registration error, thus the indentation of the gelatin phantom is not measurable during the contact.

As reported in Table III, the amplitude variability computed among users on the force $\left(\mathbf{V}_{\mathrm{F}}\right)$ and penetration depth $\left(\mathbf{V}_{\mathrm{P}}\right)$ signals during the holding phases in the assisted task executions (RMS error below $0.02 \mathrm{~N}$ and $0.1 \mathrm{~mm}$ respectively) resulted significantly reduced with respect to the freehand modality (RMS error above $0.04 \mathrm{~N}$ and $0.6 \mathrm{~mm}$ respectively).

\section{DISCUSSION}

In order to enhance the human-robot cooperation during targeting tasks on soft tissues in surgery, such as during brain cortex stimulation procedures in open-skull neurosurgery, a torque controller with non-linear force feedback (FFE) is proposed. The controller provides enhanced haptic perception 
to the operator when the surgical tool is in contact with the soft tissue. In fact, such contact forces between the surgical tool and the soft tissues, e.g. brain (peak forces below $0.2 \mathrm{~N}$ [25]) and/or liver (peak forces below 1N [26]), are too small to be perceived by the user during the robotic guidance [27], due to the residual friction of the manipulator. Differently from vitro-retinal surgery [13] and/or needle insertion procedures [8], in which the accuracy requirements are very high demanding in a constrained and limited working space, position-based force-to-motion control schemas [14] may not be the best suitable solution to provide the manipulator with the high compliance desirable during wide motion targeting tasks, e.g. brain cortex stimulation. The proposed approach is based on a torque-based cooperative control with a force feedback augmentation strategy, to augment the surgeon's ability in performing a safe and stable contact with the soft tissue.

To comparatively assess the performance of the proposed FFE controller in a controlled and repeatable setup, tests were performed using gelatin phantoms at different concentrations $(8-16 \%)$, where the $8 \%$ concentration gelatin is reported to better represents the viscoelastic properties of the brain [30]. Strictly dependent on the tool shape and geometry [28], the puncturing forces measured during the gelatin characterization protocol are consistent with the ranges reported in [25][26], i.e. increasing with respect to gelatin concentration and to the tool insertion velocity. Accordingly, a safety force criterion was considered to evaluate the tissue damage during indentation, depending on the concentration of each gelatin phantom $(0.4 \mathrm{~N}$ with $8 \%, 0.7 \mathrm{~N}$ with $12 \%, 1 \mathrm{~N}$ with $16 \%$ ).

The FFE controller proved to significantly reduce the amount of forces applied to the tissue during indentation with respect to both the freehand execution and the robotic

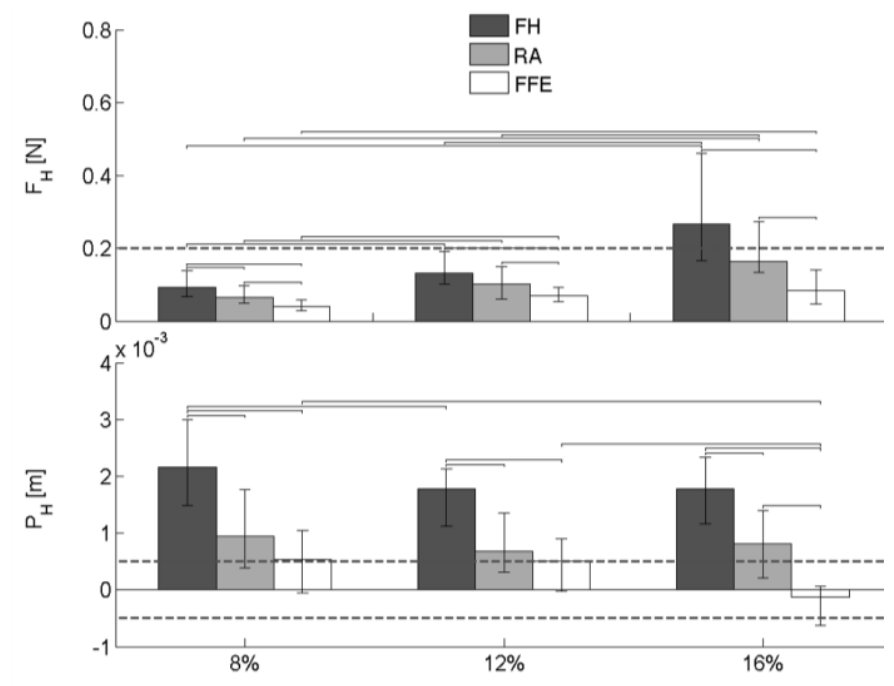

Figure 5. Evaluation of the equilibrium force $\mathbf{F}_{\mathrm{H}}$ and penetration $\mathbf{P}_{\mathrm{H}}$ indexes using the three task execution modalities (FH, RA, FFE) and the three different concentration gelatine phantoms $(8 \%, 12 \%, 16 \%)$, for all trials of the 6 nonexpert users. Vertical bars represent median values and quartiles $(25 \%$ and $75 \%$ ) of performance index population. Horizontal lines represent statistically significant differences as determined in intra-group and inter-group comparisons (Kruskal-Wallis test, $\mathrm{p}<0.05$ ) with Bonferroni-Holms correction. Also, the force safety margin used to compute the optimal FFE control parameters (dot line, above graph) and the surface registration error threshold (dot lines, graph below) are reported. assistance without force enhancement, as shown by the equilibrium force index $\mathbf{F}_{\mathrm{H}}$ (Fig. 5). During FH and RA task executions, the users reach the contact equilibrium using pure visual feedback information, as proved by comparable penetration depths (mean $\mathbf{P}_{\mathrm{H}} \approx 2 \mathrm{~mm}$ in $\mathrm{FH}$ and mean $\mathbf{P}_{\mathrm{H}} \approx 1 \mathrm{~mm}$ in RA) among different concentration gelatin phantoms. Conversely, during FFE task execution, the effect of the haptic feedback provided to the user is shown by comparable tissue interaction forces at the equilibrium $\left(\mathbf{F}_{\mathrm{H}} \approx 0.1 \mathrm{~N}\right)$ for the $12 \%$ and $16 \%$ gelatin phantoms. In fact, the indentation level during contact with the $16 \%$ gelatin is reduced below the registration accuracy of the phantom's surface $(0.5 \mathrm{~mm})$, thus it can be considered negligible. It has to be noted that the penetration depth index is not measurable in this case, but the actual tissue contact is guaranteed by a positive median equilibrium force. Although the median equilibrium forces applied by the users in each experimental condition were below the identified safety levels for each task execution modes and no tissue penetration were registered, the indentation with FFE was significantly reduced.

Even if the execution time in RA and FFE resulted slightly increased with respect to FH execution modality (as shown in Table II), the use of a cooperative robotic assistant was confirmed to allow for a more stable contact with respect to freehand task execution, providing hand tremor rejection due to the reduced bandwidth of the manipulator. The estimate of the hand tremor during contact $\left(\mathbf{V}_{\mathrm{P}}>0.6 \mathrm{~mm}\right.$, as reported in Table III) was greater than other studies [33], but it has to be noted that the users were asked to perform the targeting tasks while standing and without arm support, in order to be comparable to the tasks execution with robotic assistance.

It has to be noted that the experimental validation was performed on static soft tissue phantoms, but in the real surgical scenario predictable and/or unpredictable motions of the organ of interest can occur [4][5]. In this condition, the FFE controller is expected to increase the safety of the surgical procedure, acting as a virtual boundary during unwanted contact and preventing tissue penetration. Tests under dynamic condition would be performed in the future to evaluate this feature. Moreover, the non-linear characteristic of the FFE controller was optimally tuned based on a predefined safety margin $(50 \%$ of the critical puncturing force), which strictly depends on the mechanical characteristics of the specific tissue, and set constant during the contact. In order to generalize the approach to softer/harder tissues, an adaptive criterion based on the online estimate of the tissue stiffness should be added to the FFE controller.

\section{CONCLUSION}

Non-linear force feedback torque control was presented and experimentally evaluated on a flexible joint redundant manipulator to provide enhanced haptic perception during cooperatively assisted targeting tasks in surgery. The FFE controller was proved to allow for a stable, i.e. millimeter free-hand tremor rejection during the holding phase, and safe, i.e. $50 \%$ reduction of the indentation level, contact with the tissues. Future work will address the evaluation of the FFE controller with a pool of expert surgeons on a realistic brain phantom model, in order to exploit the possibility of realizing 
safety features for cooperatively-controlled interactions with moving organs.

\section{ACKNOWLEDGMENT}

The authors would like to thank Prof. F. Rodriguez y Baena and Prof. D. Prattichizzo for kindly provide the F/T sensors used in the experimental setup. This work was founded by the FP7 ACTIVE project (FP7-ICT-2009-6270460) and by the Scuola Interpolitecnica di Dottorato.

\section{REFERENCES}

[1] S. M. Singer, and D. L. Akin, "A Survey of Quantitative Team Performance Metrics for Human-Robot Collaboration," in International Conference on Environmental Systems, vol. 5248, no. AIAA, 2011

[2] A. Szelenyi, L. Bello, H. Duffau, E. Fava, G. C Feigl, M. Galanda, G. Neuloh, F. Signorelli, F. Sala and Workgroup for Intraoperative Management in Low-Grade Glioma Surgery within the European LowGrade Glioma Network, "Intraoperative electrical stimulation in awake craniotomy: methodological aspects of current practice," in Neurosurgical focus, vol. 28, pp. E7, Feb, 2010.

[3] N. Jarrassé, J. Paik, V. Pasqui, and G. Morel, "How can human motion prediction increase transparency?," in Robotics and Automation, 2008. ICRA 2008. IEEE International Conference on., pp. 2134-2139, 2008

[4] E. De Momi, C. A. de Oliveira Faria, O. Sadowsky, E. Bicho, G. Ferrigno, L. Joskowiz, M. Shoham, and R. Vivanti, "Validation of a stereo camera system to quantify brain deformation due to breathing and pulsatility," in Medical Physics, accepted.

[5] D. De Lorenzo, E. De Momi, L. Conti, E. Votta, M. Riva, E. Fava, L. Bello, and G. Ferrigno, "Intraoperative forces and moments analysis on patient head clamp during awake brain surgery," in Medical \& Biological Engineering \& Computing, vol. 51, no. 3, pp. 331-341, 2013.

[6] A. M. Okamura, "Haptic feedback in robot-assisted minimally invasive surgery," in Current opinion in urology, vol. 19, no. 1, pp. 102-107, 2009.

[7] T. Yamamoto, N. Abolhassani, S. Jung, A. M. Okamura, and T. N. Judkins, "Augmented reality and haptic interfaces for robot-assisted surgery," in The International Journal of Medical Robotics and Computer Assisted Surgery, vol. 8.1, pp. 45-56, 2012.

[8] D. De Lorenzo, Y. Koseki, E. De Momi, K. Chinzei, and A. Okamura, "Coaxial needle insertion assistant with enhanced force feedback," in Biomedical Engineering, IEEE Transactions on, vol. 60, no. 2, pp. 379389, 2013.

[9] M. M. Dalvand, B. Shirinzadeh, S. Nahavandi, and J. Smith, "Effects of realistic force feedback in a robotic assisted minimally invasive surgery system," in Minimally Invasive Therapy \& Allied Technologies, vol. 23, no. 3, pp. 127-135, June 2014.

[10] C. R. Wagner and R. D. Howe, "Force feedback benefit depends on experience in multiple degree of freedom robotic surgery task," in Robotics, IEEE Transactions, vol. 23, no. 6, pp. 1235-1240, Dec. 2007.

[11] B. Demi, T. Ortmaier, and U. Seibold, "The touch and feel in minimally invasive surgery," in Haptic Audio Visual Environments and their Applications, IEEE International Workshop on., IEEE, 2005.

[12] H. Mayer, I. Nagy, A. Knoll, E. Braun, R. Bauernschmitt, and R. Lange, "Haptic feedback in a telepresence system for endoscopic heart surgery," in MIT Presence, Teleoperators and Virtual Environments, vol. 16, no. 5, pp. 459-470, 2007.

[13] A. Uneri, M. A. Balicki, J. Handa, P. Gehlbach, R. H. Taylor, and I. Iordachita, "New Steady-Hand Eye Robot with micro-force sensing for vitreoretinal surgery," in Proceedings of the IEEE/RAS-EMBS International Conference on Biomedical Robotics and Biomechatronics, pp. 814-819, 2010.

[14] D. A. Lawrence, "Impedance Control stability properties in common implementation," in Robotics and Automation, 1988. Proceedings., 1988 IEEE International Conference on, 1185-1190, 1988.

[15] B. Davies, M. Jakopec, S. J. Harris, F. Rodriguez y Baena, A. Barrett, A. Evangelidis, P. Gomes, J. Henckel, and J. Cobb, "Active-constraint robotics for surgery," in Proceedings of the IEEE, vol. 94, no. 9, pp. 1696-1704, 2006.
[16] A. Albu-Schaffer, C. Ott, and G. Hirzinger, "A passivity based cartesian impedance controller for flexible joint robots - part ii: fullstate feedback, impedance design and experiments," in Robotics and Automation, 2004. Proceedings. ICRA'04. 2004 IEEE International Conference on., vol. 3, pp. 2666-2672, 2004.

[17] X. He, M. Balicki, P. Gehlbach, J. Handa, R. Taylor, and I. Iordachita, "A Multi-Function Force Sensing Instrument with Variable Admittance Robot Control in Retinal Microsurgery," in Robotics and Automation (ICRA), 2014 IEEE International Conference on., Hongkong, China, May 31-June 7, 2014.

[18] P.J. Berkelman, L. L. Whitcomb, R. H. Taylor, and P. Jensen, "A miniature instrument tip force sensor for robot/human cooperative microsurgical manipulation with enhanced force feedback," in Medical Image Computing and Computer-Assisted Intervention-MICCAI 2000, Springer Berlin Heidelberg, pp. 897-906, 2000.

[19] P. Kazanzides, J. Zuhars, B. Mittelstadt, and R. H. Taylor, "Force sensing and control for a surgical robot," in Robotics and Automation, 1992. Proceedings., 1992 IEEE International Conference on., pp. 612617, 1992.

[20] M. S. Erden, and B. Marić, "Assisting manual welding with robot," in Robotics and Computer-Integrated Manufacturing, vol. 27, no.4, pp. 818-828, 2011.

[21] V. Duchaine, and C. M. Gosselin, "General Model of Human-Robot Cooperation Using a Novel Velocity Based Variable Impedance Control," in EuroHaptics Conference, 2007 and Symposium on Haptic Interfaces for Virtual Environment and Teleoperator Systems. World Haptics 2007. Second Joint., pp. 446-451, 2007.

[22] E. Beretta, E. De Momi, F. Rodriguez y Baena, and G. Ferrigno, "Adaptive hands-on control for reaching and targeting tasks in surgery," in International Journal of Advanced Robotic System, 2015, vol. 12, no.50, 1-9, doi: 10.5772/60130.

[23] A. M. Okamura, "Methods for haptic feedback in teleoperated robotassisted surgery," in Industrial Robot: An International Journal, vol. 31, no. 6, pp. 499-508, 2004.

[24] X. He, B. Marcin, P. Gehlbach, J. Handa, R. Taylor, and I. Iordachita, "A novel dual force sensing instrument with cooperative robotic assistant for vitreoretinal surgery," in Robotics and Automation (ICRA), 2013 IEEE International Conference on., pp. 213-218, 2013.

[25] K. Miller, C. Kiyoyuki, O. Girma, and B. Piotr, "Mechanical properties of brain tissue in-vivo: experiment and computer simulation," in Journal of Biomechanics, vol. 33, no. 11, 1369-1376, 2000.

[26] B. Maurin, L. Barbe, B. Bayle, P. Zanne, J. Gangloff, M. De Mathelin, A. Gangi, L. Soler. And A. Forgione, "In vivo study of forces during needle insertions," in Proceedings of the medical robotics, navigation and visualisation scientific workshop., pp. 415-422, March 2004.

[27] P. Marayong, G. D. Hager, and A. M. Okamura, "Effect of hand dynamics on virtual fixtures for compliant human-machine interfaces," in Haptic Interfaces for Virtual Environment and Teleoperator Systems, 2006 14th Symposium on., pp. 109-115, 2006.

[28] N. Abolhassani, P. Rajni, and M. Mehrdad, "Needle insertion into soft tissue: A survey" in Medical Engineering \& Physics, vol. 29, no. 4, pp. 413-431, 2007.

[29] D. Stein, H. Monnich, J. Raczkowsky, and H. Worn, "Visual servoing with an optical tracking system and a lightweight robot for laser osteotomy," in Control and Automation, 2009. ICCA 2009. IEEE International Conference on., 2009, pp. 1896-1900, 2009.

[30] D. De Lorenzo, Y. Koseki, E. De Momi, K. Chinzei, and A.Okamura. "Experimental evaluation of a coaxial needle insertion assistant with enhanced force feedback," in IEEE Engineering in Medicine and Biology Society, 2011 33rd Annual international Conference of, pp. 3447-3450, 2011.

[31] D. Kubus, T. Kroger, and F. M. Wahl, "On-line rigid object recognition and pose estimation based on inertial parameters," in Intelligent Robots and Systems, 2007. IROS 2007. IEEE/RSJ International Conference on., 2007.

[32] F. Janabi-Sharifi, V. Hayward, and C. S. J. Chen, "Discrete-time adaptive windowing for velocity estimation," in Control Systems Technology, IEEE Transactions on, vol. 8, no. 6, pp. 1003-1009, 2000.

[33] R. A. MacLachlan, B. C. Becker, J. C. Tabares, G. W. Podnar, L. A. Lobes, Jr., and C. N. Riviere, "Micron: An actively stabilized handheld tool for microsurgery," in Robotics, IEEE Transactions on, vol. 28, no.1, pp. 195-212, Feb. 2012. 